\title{
Estrategia formativa para mitigar la violencia escolar en perspectiva de derechos humanos
}

\section{Training strategy to mitigate school violence from a human rigths perspective}

Gerzon Yair Calle-Álvarez ${ }^{1}$ - Diego Alejandro Ocampo-Zapata² - Erika María Franco-Coteiro³ - Lucy Delvi Rivera-Gil ${ }^{3}$

http://orcid.org/0000-0002-4083-6051 - Universidad de Antioquia, Medellín, Colombia iD http://orcid.org/0000-0002-5286-0964 ${ }^{3}$ iD http://orcid.org/0000-0002-8819-2996

UniversidadCatólicadeOriente,Medellín,Colombia

Resumen

Objetivo: La finalidad de este estudio es presentar una estrategia formativa que busca mitigar la violencia escolar desde la perspectiva de los derechos humanos.

Método: se realizó una investigación de corte cualitativo en el que participaron 72 estudiantes de la educación media de una institución educativa pública de la ciudad de Medellín.

Resultados: se encontró que para lograr mitigar los problemas de violencia escolar en la escuela se deben buscar estrategias que convoque a los diferentes integrantes de las comunidades educativas.

Conclusiones: se logró elaborar una estrategia formativa en la institución educativa para mitigar las diversas formas de violencia escolar en el marco de los derechos humanos. La propuesta se centró en el tema de la discriminación presente en esta institución, por cuanto el trabajo de diagnóstico realizado con los estudiantes puso en evidencia que esta era una de las prácticas escolares consideradas como normales.

Palabras clave: Derechos humanos, Escuela, Estrategia de prevención, Violencia escolar.

Abstract

Objective: The purpose of this study is to present a strategy that seeks to mitigate school violence from the perspective of human rights.

Method: a qualitative research was carried out in which 72 middle school students of a public educational institution of the city of Medellín participated.

Results: it was found that to achieve it. The problems of school violence in the school should be sought strategies that convene the different members of the educational communities.

Conclusions: a formative strategy was developed in the educational institution to mitigate the different forms of school violence within the framework of human rights. The proposal focused on the issue of discrimination present in this institution, since the diagnostic work carried out with the students showed that this was one of the school practices considered normal.

Key words: Human rights, School, Prevention strategy, School violence.
Open Access:

Editor:

Patricia Martínez Barrios Universidad Simón Bolívar

Correspondencia Gerzon Yair Calle-Álvarez gerzon.calle@udea.edu.co

Recibido: 16-12-16 Aceptado: $31-04-17$

Publicado: 01-01-18

DOI: http://dx.doi org/10.17081 eduhum.20.34.2859 


\section{INTRODUCCIÓN}

Uno de los primeros acercamientos al concepto de violencia escolar destaca que es aquella

que se produce en el marco de los vínculos propios de la comunidad educativa y en el ejercicio de los roles de quienes la conforman: padres, estudiantes, docentes, directivos. Son el producto de mecanismos institucionales que constituyen prácticas violentas y/o acentúan situaciones de violencia social. (United Nations International Children's Emergency Fund-UNICEF, 2011, p.9)

La violencia escolar es un fenómeno que se configura dentro de las instituciones educativas y que viene despertando el interés en la comunidad internacional, académica y de autoridades nacionales, departamentales, locales e institucionales, por su trascendencia, consecuencias en el desarrollo integral de los estudiantes y en el impacto que ciertos tipos de comportamientos pueden tener, a futuro, en la sociedad. Para la United Nations Educational, Scientific and Cultural Organization - UNESCO (2011), la problemática mundial está determinada por cuatro formas de violencia en la escuela: el castigo físico y psicológico, el acoso, la violencia sexual y por razones de género, y la violencia externa.

De acuerdo con Eljach (2011) la violencia escolar en Latinoamérica se presenta bajo dos escenarios específicos: a) El llevado a cabo por los profesores y directivos docentes, quienes establecen reglas que atentan contra los derechos humanos de los estudiantes y tiene sus principales manifestaciones en los castigos corporales; el maltrato emocional; en la discriminación étnica y racial y; por último, en la violencia sexual; 2) La violencia entre pares, que también tiene diversas formas de manifestarse, siendo la más relevante el bullying, que se caracteriza por el maltrato, acoso y violencia física. En cualquiera de los escenarios la violencia escolar requiere de estrategias de prevención que puedan mitigar estos comportamientos al interior de las instituciones educativas, para que se puedan crear las condiciones sociales que aporten a la formación de los estudiantes.

Además, se hace necesario considerar que algunos mecanismos de violencia escolar no son visibles en la escuela, debido a que son tan sutiles y tienen tal nivel de carga psicológica, que ocasiona en las víctimas altos niveles de presión que finalmente salen a relucir cuando ya la situación de violencia ha perdido todo su control. Romero (2012) pone en evidencia el costo humano, 
económico y social de la violencia escolar en Latinoamérica, que es muy alto ya que "socava los fundamentos democráticos de una sociedad, su vida colectiva y la calidad de su desarrollo" (p.11).

Villalba (2016) realizó una revisión teórico-práctica sobre la violencia escolar y las posibles interpretaciones que se le puede dar desde las instituciones educativas. Dentro de las conclusiones se presenta que las instituciones están llamadas a ver los conflictos como oportunidades para fortalecer la convivencia escolar e inherente al desarrollo social; por otra parte, es prioritario un enfoque de educación multidimensional que incluya las habilidades del saber, hacer, al tiempo que las del ser y el convivir.

En los últimos años en el contexto colombiano el tema de la violencia escolar ha tomado mucha fuerza, un ejemplo de ello, es la Ley 1620 de 2013, denominada "Ley de Convivencia Escolar" y el Decreto 1695 de 2013. Los cuales tienen dentro de sus orientaciones fomentar la convivencia sana dentro de los establecimientos educativos, buscando mitigar el acoso escolar, ciberbullying, el maltrato a los derechos humanos y cualquier conducta que perturbe la integridad de los estudiantes. Además, el Ministerio de Educación Nacional MEN (2013) publicó la Guía Pedagógica 39 para la Convivencia Escolar, que brinda herramientas para la adopción de la Ley 1620 de 2013 y el Decreto 1695 de 2013 dentro de las instituciones educativas. Otra de las acciones para el manejo de la violencia escolar proviene de la Policía Nacional de Colombia (2010) a través del Programa "Delinquir no paga", con el cual se buscan

adelantar acciones orientadas a evitar la incursión de los jóvenes de Medellín en actividades fuera de la norma social y además promover en ellos la autorregulación, la cultura y la convivencia ciudadana, partiendo del respeto por sí mismo y el respeto de los demás. (p.3)

En el contexto de ciudad de Medellín, se han realizado diversas investigaciones sobre el tema de la violencia escolar, asumiendo diversas miradas sobre el problema: el psicológico, el social, la familia. En el estudio de Echeverri, Gutiérrez, Ramírez \& Morales (2014) se realizó una revisión documental sobre los conceptos de violencias escolares en bases de datos, con el propósito de determinar los discursos y prácticas relativas a la violencia escolar en el Colegio Parroquial Emaús, ubicado en la ciudad de Medellín. Dentro de las conclusiones se manifiesta la necesidad de hacer una diferenciación sobre las diversas formas de violencias escolares, así como su identificaciónconforme 
a las disciplinas que lo están investigando, ya que ello coadyuva a desarrollar estrategias integrales para su superación o mitigación.

Tobón (2014) realizó una investigación con el propósito de identificar las representaciones sociales sobre la violencia escolar presente en una institución educativa del municipio de Medellín, bajo la tríada convivencia, conflicto y violencia escolar. Dentro de las conclusiones se subraya cómo las diversas formas de violencia presentes tanto en el colegio como en su entorno social, ponen en evidencia una afectación a la vida cotidiana de los estudiantes y sus familias, generando como principal efecto una permeabilización de las relaciones entre los diversos actores presentes en la institución educativa. Esto implica llevar a cabo procesos de intervención más profundos, pues no se pueden resolver con actividades escolares formales, sino con acciones conjuntas que ayuden a minimizar los riesgos de violencia escolar.

Otra de las contribuciones para la comprensión de la problemática en Medellín es la realizada por Gil (2013) quien hace un estudio sobre la violencia en este municipio, particularmente en el periodo 1993-2013, el cual se encuentra enmarcado en la profunda influencia de los grupos armados ilegales, quienes aprovechan las graves condiciones socioeconómicas y familiares de los jóvenes para adoctrinarlos en aspectos de la guerra y la lucha contrainsurgente, generando un clima de violencia que se traslada a todos los escenarios como los colegios, las comunidades y la forma como se asumen los conflictos y las diferencias.

En un trabajo realizado por González, Mariaca \& Arias (2014) sobre el bullying en Medellín, se encontró que las formas más frecuentes de agresión entre estudiantes son los golpes (10,6\%), insultos $(6,07 \%)$, burlas $(6,69 \%)$, y apodos $(5,45 \%)$, significando con ello el surgimiento de conflictos y tensiones entre los estudiantes, pues se empiezan a construir fronteras invisibles que agravan más el problema, pues la violencia se torna como una forma natural de interacción.

Desde el punto de vista práctico, la presente investigación se orientó en la intención por llevar a cabo un mayor compromiso de los profesionales por aplicar los fundamentos teóricos aprendidos, de forma tal que permita cambios en las diversas prácticas sociales, específicamente en la relacionada con la problemática de la violencia escolar en el entorno educativo. Para la Organización de las Naciones Unidas - ONU (2014) es claro que el proceso de enseñanza en derechos humanos requiere de la participación activa de 
los docentes, quienes tienen la tarea de socializar las experiencias relacionadas con el manejo de los derechos humanos en la práctica educativa, así como del tratamiento dado a los casos de violencia escolar, para lo cual deben establecer redes de apoyo entre docentes, profesionales que aporten con su conocimiento y experiencias, organizaciones o grupos de derechos humanos y asociaciones profesionales interesadas en el abordaje de estas problemáticas.

Es importante señalar que las instituciones educativas son las llamadas a comprometerse en la recuperación del terreno perdido frente a las diversas formas de violencia escolar, buscando con ello generar nuevos espacios de convivencia y mejoramiento de los derechos humanos en ambientes escolares, de tal forma que trasciendan en los contextos escolares y las comunidades circundantes. Frente a estos espacios Albor Chadid y Villamil López (2012) y Cataño (2014) subrayan que uno de los problemas es que existe un desconocimiento sobre las características de dichas prácticas de convivencia, generando que se incurra en fallas al momento de elaborar los planes o proyectos enfocados a mitigar la violencia escolar.

En la búsqueda de experiencias de implementación de estrategias sobre convivencia en Derechos Humanos, con el fin de mitigar la violencia en la escuela, se resalta la experiencia implementada en las escuelas de Bucaramanga. Esta investigación se desarrolla desde la necesidad de intervenir un complejo problema que se vive en las escuelas, consistente en el aumento de los conflictos y las violencias en los que los sujetos activos son los menores de edad (Correa, Jaimes, Cardozo \& Mantilla, 2013). La Investigación muestra cómo desde un estudio sociojurídico, se establecen mecanismos de orientación para formar en competencias ciudadanas desde la escuela, teniendo como fundamentos la teoría de la civilidad, la cual es vista a partir de su bifurcación entre civilismo y civismo. Esta investigación permitió después de la construcción teórica en competencias ciudadanas, implementar un Centro de Orientación Interdisciplinario para formar tales competencias en la escuela.

Albor Chadid \& Villamil López (2012) realizaron una investigación que tenía dentro de sus objetivos identificar las prácticas de aula que realizaban los docentes de los grados de séptimo y octavo, de dos instituciones educativas, de las ciudades de Barranquilla y Girón, para mitigar la violencia escolar. Dentro de las conclusiones se encuentran: la violencia trasciende de lo personal a lo interpersonal y se manifiesta a través de agresiones físicas, verbales, psicológicas y, en menor escala, lo sexual. Las condiciones de las instituciones han 
generado que los profesores no se motiven a generar acciones acertadas que permitan la mediación de los conflictos, además, se observa que los profesores no cuentan con las herramientas teórico y prácticas para generar espacios de diálogos con los estudiantes.

El presente artículo tiene como objetivo presentar los principales elementos a considerar en la elaboración de una estrategia de formación que permita mitigar las diversas formas de violencia escolar en perspectiva de los derechos humanos dentro de las instituciones educativas, basado en el enfoque establecido por la UNESCO (2012), el cual plantea que los derechos humanos son ante todo procesos vivenciales que permiten "el conocimiento, las capacidades y las actitudes para promover comportamientos respetuosos con los derechos humanos" (p.2).

\section{METODOLOGÍA}

La investigación en educación se encuentra orientada a la producción y construcción de conocimiento en comunidades académicas, que sin duda contribuye no solo en la formación cualitativa del maestro, sino en la capacidad de mejorar los procesos o prácticas con los estudiantes. Martínez (2005) precisa que la finalidad de la investigación en educación consiste en conocer con cierta precisión "una determinada realidad educativa, sus características y funcionamiento, así como la relación que existe entre los elementos que la configuran" (p.18). Con ello, es posible que se puedan hacer valoraciones o diagnósticos sobre la realidad analizada, a la vez que configurar escenarios futuros o posibles efectos que se pueden producir.

El marco metodológico del presente trabajo de investigación está dado por un enfoque cualitativo. El desarrollo del proceso de investigación implicó varias tareas: 1) reconocimiento de las diversas formas de violencia escolar dentro de la institución educativa buscando un acercamiento a las realidades de los estudiantes; 2) identificar las estrategias didácticas que se podían aplicar e implementar dentro de la institución educativa que pudieran repercutir en la mitigación de la violencia escolar; 3) evaluar la estrategia de formación con los estudiantes. El alcance de este proceso de intervención y evaluación está orientado a generar una mayor concientización por parte de los integrantes de la institución educativa sobre las formas en que se materializa la violencia escolar y las alternativas para mitigarlas, favoreciendo los procesos de convivencia desde los Derechos Humanos.

La investigación se desarrolló en una institución educativa de la Comuna 
12, zona occidental, de la ciudad de Medellín, con 72 estudiantes de grado décimo: 41 mujeres y 31 hombres, perteneciente a los estratos socioeconómicos dos y tres, con edades que oscilan entre los 15 y 17 años, pertenecientes al grado décimo.

Se aplicó el método de muestreo por conveniencia, donde el investigador decidió qué individuos de la población pasan a formar parte de la muestra en función de la disponibilidad de los mismos, el tiempo que lleva en la institución educativa (más de dos años). Para seleccionar esta muestra se definió la unidad de análisis, se pudo determinar que la población más vulnerable frente al problema de la violencia escolar y vulneración de los derechos humanos era la educación media, además de rastreos de otras fuentes como, ficha de seguimiento comportamental, informes del comité de convivencia escolar y reporte de estudiantes sancionados, brindados por la coordinación de convivencia, se constató que efectivamente estos eran los grupos donde más se presenta este fenómeno de violencia escolar y de desconocimiento de derechos en las relaciones de convivencia.

Con el fin de construir la estrategia de formación se conformó un grupo de discusión que estuvo integrado por un coordinador de convivencia de la institución, un profesor de la educación media, un profesional encargado del equipo de convivencia, cuatro estudiantes líderes de los últimos grados (décimo y undécimo) y uno de los investigadores del presente estudio. El propósito de este grupo de discusión era actuar como líderes durante el proceso de diseño e implementación.

Con el fin de llevar a cabo una evaluación de la propuesta para mitigar la violencia escolar, se diseñó una entrevista, en la que participaron 12 estudiantes de grado décimo. La entrevista estaba orientada por las siguientes preguntas: 1) ¿cómo le pareció la "Jornada escolar por la convivencia y el rechazo a todas las formas de discriminación”?; 2) ¿De qué forma pueden contribuir estas jornadas para bajar la violencia escolar en nuestra institución educativa?; 3) ¿Ha evidenciado cambios en sus compañeros después de la jornada que se realizó sobre los derechos humanos y la violencia escolar?; 4) Si se presenta una situación de agresión física o verbal en el colegio, ¿usted qué haría?; 5) ¿De qué forma se comprometería usted para mejorar el respeto por los derechos humanos?

\section{RESULTADOS Y DISCUSIÓN}

Los resultados que se presentan a continuación buscan responder al objetivo 
de presentar una estrategia de formación que busque mitigar la violencia escolar desde la perspectiva de los derechos humanos. Para ello, se partió de las realidades institucionales, se procedió a construir la propuesta de intervención con algunos integrantes de la comunidad educativa, se implementó la estrategia y finalmente se evalúo desde la percepción de los estudiantes que participaron de la estrategia.

El diseño de la estrategia formativa implicó la comprensión del contexto escolar donde se llevaría a cabo la implementación. Así, se encontró una institución donde los estudiantes manifestaban actitudes de violencia física y verbal entre ellos, presunciones de robos y maltrato psicológico. Además, se identificaron actividades de bullying y ciberbullying. Partiendo de esta realidad se decidió crear una estrategia por fases que fueran integrando a los diversos actores de la comunidad educativa.

De lo analizado, la estrategia de formación planteada corresponde a la realización de una campaña de sensibilización sobre las diversas formas de discriminación presentes en la institución educativa y su relación con la violencia escolar. Esta campaña se inició con una jornada escolar para la identificación de estas formas de discriminación y su materialización en conductas de estudiantes, docentes y directivos.

De igual forma, al ser una problemática ligada a la vulneración de los derechos fundamentales de los estudiantes, la orientación que se estableció para esta jornada escolar fue el planteado por la UNESCO (2011) como un planteamiento holístico, cuyo fundamento está en el enfoque pedagógico basado en los derechos humanos, por cuanto permite que se fomente "el acceso a la enseñanza y la participación en ella, pues favorece la integración, la diversidad, la igualdad de oportunidades y la no discriminación" (p.8).

Vale decir que, de las diez esferas de acción propuestas por la UNESCO, se tomó como eje principal de la jornada escolar "Lograr que sus estudiantes se involucren con usted (el maestro) en la prevención de la violencia” (UNESCO, 2011, p.15). Este tipo de acciones se enmarcan dentro de la posibilidad de llevar a cabo procesos de enseñanza en materia de derechos humanos, los cuales se pueden aplicar en escenarios diversos como en el uso de historia, debates, actividades teatrales, juegos y actividades deportivas que logren mitigar y sensibilizar sobre los efectos que tiene la discriminación en el ámbito escolar.

El proceso de elaboración de la propuesta y su implementación se llevó a 
partir de unas fases definidas por el equipo de profesionales encargados del manejo de la convivencia escolar en la institución educativa. Estas fases para la realización de la jornada escolar fueron las siguientes:

Fase 1. Creación del equipo coordinador. El equipo coordinador estuvo compuesto por un representante de los docentes, un profesional encargado del equipo de convivencia, un representante de los directivos docentes, 4 estudiantes líderes de los últimos grados $\left(10^{\circ}\right.$ y $\left.11^{\circ}\right)$ y uno de los investigadores del presente estudio, quien a la vez hacía parte del equipo de maestros de la institución educativa.

Una vez conformado el equipo coordinador, se definieron los objetivos de la jornada escolar: 1) Identificar las principales formas de discriminación presentes en la institución educativa; 2) Reflexionar sobre cuáles son los derechos humanos que más se vulneran al interior de la institución educativa; 3) Llegar a unos acuerdos mínimos para el tratamiento de la violencia escolar en la institución educativa, desde la mirada de los derechos humanos.

El lema dado a la jornada escolar fue "La discriminación afecta y vulnera nuestros derechos y la dignidad de la persona". Definidos los objetivos y el lema, se procedió a crear los comités de preparación de la jornada, estableciendo tres de ellos como son el de maestros, estudiantes y directivos de la institución, para esta parte, los integrantes del equipo coordinador se convertían en líderes de cada uno de los comités

Fase 2. Definición de las actividades a desarrollar en la "Jornada escolar por la convivencia y el rechazo a todas las formas de discriminación”. Estas actividades fueron trabajadas por cada uno de los comités, de forma independiente, estableciendo que cada una de estas actividades fuera desarrollada en un máximo de dos horas. Dichas actividades programadas finalmente fueron socializadas y consolidadas por el equipo coordinador. Las actividades se fundamentaron en debates, talleres y ágape comunitario.

Fase 3. Realización de la "Jornada escolar por la convivencia y el rechazo a todas las formas de discriminación”. Esta jornada de sensibilización estuvo marcada por diversas actividades, centradas en profundizar sobre el valor de los derechos humanos y el impacto que tienen las diversas formas de discriminación. Una de estas actividades consistió en la realización de un debate con los estudiantes de los últimos grados, cuyo objetivo fue sensibilizar sobre la importancia de su liderazgo sobre los demás estudiantes y el papel que tiene el respeto por los derechos humanos como mecanismo para fortalecer 
dicho liderazgo, sin que ello implique escenarios de discriminación. Como un insumo para la memoria de la jornada, se desarrolló un trabajo por grupos en los cuales los estudiantes plasmaron los principales acuerdos para llevar a cabo un liderazgo basado en el respeto por los derechos humanos.

Otra de las actividades desarrolladas correspondió a la realización de acciones de sensibilización y concientización sobre las diversas formas de discriminación, labor de la cual fueron responsables un grupo conformado por docentes y estudiantes, quienes desarrollaron talleres al interior de los salones, con la participación de todos los grados, en donde los estudiantes pudieron expresar cuáles eran los principales problemas de discriminación que vivía el colegio y cuáles se evidenciaban en nuestra ciudad. Los resultados mostraron que el tema de la discriminación por género era la más relevante, seguida de la discriminación por saberes, en la medida que había situaciones de exclusión o de rechazo hacia aquellos estudiantes que presentan dificultades de aprendizaje.

Otra actividad destacada correspondió a la desarrollada por los estudiantes de $10^{\circ}$, los cuales prepararon un ágape comunitario, en el que se desarrolló un diálogo abierto sobre cuáles son las principales conductas que asumimos en la escuela, en la calle y en la familia que fomentan mecanismos de discriminación. Al final de esta actividad se plantearon unos acuerdos sobre conductas favorables a los derechos humanos.

Finalizando la jornada se realizó una actividad conjunta de toda la comunidad educativa en la que se socializaron los resultados de los acuerdos a los que llegaron los diferentes grupos de trabajo, siendo los más relevantes los siguientes:

- Los integrantes de la institución educativa deben tener mayor conciencia de sus actos, pues con algunas actitudes y palabras generan situaciones de discriminación que afectan de forma profunda a las otras personas.

- Los apodos corresponden a una de las formas más evidentes de discriminación, pues estos no buscan enaltecer a la persona, sino ponerla en ridículo y excluirla de los demás. Fue tarea de todos eliminar los apodos a los que se encuentran sometidos algunos estudiantes de la institución.

- Las burlas por errores de los demás muestran la falta de comprensión sobre el impacto y afectación que tienen estas actitudes sobre la 
persona, pues lleva a que se sientan inferiores y no rindan en las actividades académicas como en las propias de recreación en los momentos de descanso.

- Las actitudes positivas de respeto por los compañeros de salón, se deben ampliar a los demás miembros de la comunidad educativa, a la familia y a la comunidad en general.

- El hecho de que algunos estudiantes tengan mayores habilidades para aprender no significa que se deban marginar a aquellos que presentan dificultades, esta es otra forma de discriminar.

- Se debe procurar llevar a cabo un trato igualitario entre hombres y mujeres, porque hay actitudes y palabras, en las cuales se les está maltratando a las mujeres por su condición de género.

El diseñar e implementar una estrategia formativa para mitigar la violencia escolar requiere definir los roles de cada uno de los integrantes de la comunidad educativa para lograr el objetivo de una convivencia en Derechos Humanos.

a) Directivos: favoreciendo y coordinando el desarrollo de las actividades propuestas dentro de la estrategia. Apoyando el desarrollo de los procesos.

b) Docentes: a través de la formación y de la intervención de las problemáticas desde una mirada distinta a lo punitivo para favorecer la convivencia democrática y la cultura de paz. Comprometiéndose con la capacitación en acciones de mediación escolar y solución de conflictos.

c) Estudiantes: con trabajos en el aula promoviendo una cultura de igualdad y el desarrollo de habilidades sociales y competencias emocionales, así como en el establecimiento de normas de convivencia a través del diálogo y, la conversación dadas y reguladas con los propios estudiantes. También por medio de líderes mediadores que tendrán formación en solución de conflictos, para procurar una regulación entre pares y de esta manera se entiendan las relaciones como convivencia y no como imposiciones disciplinarias.

d) Institución educativa: insertando en el PEI, la estrategia desde un enfoque transversal que favorezca el trabajo desde todas las áreas del conocimiento y permita desde el modelo Pedagógico Institucional, alcanzar los objetivos misionales y visionales. Las líneas de trabajo fundamentales en esta transversalidad serán desde la cátedra de Cívica y Urbanidad, la Formación Ciudadana, la Ética, la Cátedra para la Paz. 
e) Familia: ampliando la participación, para establecer y articular valores y normas de convivencias compatibles en la escuela y el hogar, favoreciendo un acompañamiento permanente en la educación y formación de sus hijos.

Una vez finalizada la "Jornada escolar por la convivencia y el rechazo a todas las formas de discriminación", se procedió a aplicar una entrevista a los estudiantes, a fin de evaluar si la estrategia pedagógica logró el proceso de sensibilización frente a la discriminación y su efecto en minimizar el nivel de violencia escolar en la institución educativa.

Para la participación en la encuesta se seleccionaron 12 estudiantes, para ello se debieron cumplir los siguientes parámetros.

a. Estudiantes que estuvieran en la muestra seleccionada de los 72 estudiantes.

b. Estudiantes que durante la jornada manifestaron actitudes de liderazgo, objetividad, opiniones críticas y buena capacidad discursiva.

c. Se tuvo en cuenta estudiantes que hayan estado en el colegio más de tres años, debido a que estos pueden dar un registro histórico, de las diferentes campañas que se han realizado en la institución, en torno a la misma temática y el efecto que ha tenido en relación con la mitigación de la violencia escolar y así poder determinar el nivel de mitigación que se alcanzó con esta propuesta pedagógica.

Las respuestas de los estudiantes se enfocaron en su "sorpresa" ante las diversas formas en que inconscientemente se están discriminando a los estudiantes, pues señalaron que las burlas y los apodos los tomaban como algo normal en las actividades y relaciones cotidianas, pero nunca se habían puesto a reflexionar sobre los efectos que tienen en estas personas. Frentea la mirada que tuvieron de la estrategia de formación la respuesta es generalizada en que estas jornadas debían realizarse para tratar otros temas o problemas que se viven al interior de la institución, pues al ser de desarrollo libre, no sujeto a calificación alguna, permite que los estudiantes se expresen sin ningún tipo de limitaciones.

Frente a la pregunta de si ha evidenciado cambios en sus compañeros después de la jornada, pues señalan que es muy prematuro hablar de cambios, puesto que esto es un proceso de toma de conciencia y de sensibilización, luego se requiere de más actividades para que se pueda interiorizar como una práctica consciente de respeto por los demás; ya que las actitudes y conductas que 
siguieron a cada una de las actividades fueron inicialmente una primera reacción, pero no su interiorización y concientización.

Asimismo, ante la pregunta de si se presenta una situación de agresión física o verbal en el colegio, cómo actuarían, la respuesta fue variada en cuanto a intervenir, pues fueron claros en señalar que había que conocer cuáles son las causas o las razones para que dicha agresión se presente, pero que si es entre compañeros, de seguro que tratarían de calmar la situación e, inclusive, en algunos casos defenderlo. Lo dicho por los entrevistados destaca que la solución no es fácil de asumir, pues una intervención de un tercero, en caso de que esté a favor de alguno de los que se están agrediendo, puede profundizar más el problema y originarse un conflicto más difícil y de más graves consecuencias. Sí llama la atención es que la solución no pasa por los maestros o los coordinadores de disciplina y convivencia, evidenciando la existencia de una barrera entre los mecanismos de control y prevención del colegio y las conductas o soluciones que los estudiantes establecen.

Finalmente, frente a la pregunta sobre de qué forma se comprometerían para mejorar el respeto por los derechos humanos, los estudiantes plantean acciones de tipo individual y colectivo. A nivel individual, llevar a cabo un proceso de mayor concientización sobre qué se dice y se hace; para lo cual consideran urgente llevar a cabo más actividades de este tipo, sobre diversas manifestaciones de violencia escolar. A nivel colectivo rescatan el hecho de las jornadas y de las actividades que los maestros puedan desarrollar en las diferentes disciplinas para promover el respeto por los derechoshumanos.

En síntesis, el ejercicio pedagógico y académico realizado en la institución educativa, permitió que se generaran espacios de reflexión y sensibilización sobre la violencia escolar y una de sus manifestaciones más complejas y difíciles de identificar como es la discriminación, sobre todo porque el diagnóstico permitió identificar que esta era una conducta en cierta forma "normal", impidiendo que se visibilizara como una clara violación de derechos fundamentales de los estudiantes. Al ser una actividad orientada y dirigida por toda la comunidad educativa, permitió identificar intereses, perspectivas y direccionamientos sobre el manejo de la violencia escolar.

\section{CONCLUSIONES}

Las conclusiones que se presentan pretenden responder al cumplimiento del objetivo. Vale decir que se logró elaborar una estrategia formativa en la institución educativa para mitigar las diversas formas de violencia escolar 
en el marco de los derechos humanos. La propuesta se centró en el tema de la discriminación presente en esta institución, por cuanto el trabajo de diagnóstico realizado con los estudiantes puso en evidencia que esta era una de las prácticas escolares consideradas como normales.

Se destaca que existe una íntima relación entre la violencia escolar y los derechos humanos en tanto al presentarse en la escuela sucesos que afectan física o psicológicamente a niñas y niños víctimas de sus contemporáneos, ocasiona una formación en contra de los derechos humanos, significando que se creen relaciones de víctimas-victimarios, pero no de diálogo entre estudiantes.

El pensamiento crítico coadyuva en la comprensión de dichas relaciones de poder y sometimiento, por cuanto, es una invitación a que los estudiantes miren su realidad desde otra perspectiva, en el entendido que para el abusador implica que se entere que está violando derechos fundamentales de sus compañeros y por parte de la víctima que tiene derechos que puede hacerlos exigibles en cualquier momento.

Sin duda en estos casos esas relaciones deben estar acompañadas de instrumentos jurídicos, pedagógicos y didácticos que ayuden en el proceso de toma de conciencia crítica sobre el quehacer en la institución educativa. Si bien no es un campo de batalla, sin duda se están gestando ambientes de tensión frente al fenómeno de la violencia escolar, en el cual confluyen diversos factores que están incidiendo en la forma como se profundice este tipo de violencia.

Dentro de la investigación se reafirman las conclusiones que se han logrado en investigación similares previas, como, el conflicto es parte de las sociedad y debe verse como una posibilidad de fortalecer la convivencia dentro de las instituciones (Villalba, 2016); los profesores requieren herramientas pedagógicas, didácticas y sociales para trabajar y afrontar la convivencia escolar dentro del aula (Eljach, 2011; Albor Chadid \& Villamil López, 2012); algunas manifestaciones de violencia escolar en la escuela como apodos, burlas, agresiones verbales, se vienen considerando naturales por parte de los estudiantes (González, Mariaca \& Arias, 2014) lo que implica un trabajo pedagógico para desaprender estas actitudes y crear las condiciones sociales de respeto al otro.

Frente al objetivo de plantear una estrategia de formación, vale decir que la misma se logró, tomando como base una de las líneas o esferas de acción 
impulsada por la UNESCO (2011) la que permitió que se lograra involucrar a los estudiantes en procesos de prevención y sensibilización frente a la violación de derechos fundamentales en el colegio producto de la violencia en sus distintas modalidades. Esto generó la implementación y evaluación de esta estrategia formativa, cuyos resultados positivos fueron bien señalados por los estudiantes entrevistados, quienes manifestaron su sorpresa al identificar que estaban teniendo prácticas de discriminación, de la cual no eran conscientes.

La promoción de jornadas de discusión puede ser una de las herramientas prácticas para hacer visible el problema de la violencia escolar dentro de las instituciones, la cual puede ser replicada en las instituciones escolares donde se presenten problemas de convivencia, de signos de violencia que socaven los fundamentos constitucionales de defensa de los derechos humanos.

Como recomendación para futuras investigaciones se plantea que el campo de los derechos humanos como de los escenarios de vulneración son complejos y extensos, por lo que se debe establecer procesos de investigación teórico-prácticas que asuman uno a uno los problemas presentes. Igualmente, al ser esta propuesta el resultado de una jornada de discusión, es evidente que la institución educativa participante debe implementar un proyecto de carácter institucional y transversal que permita tratar con mayor integridad el problema de la violencia escolar, a fin de que se logre mitigar con actividades dinámicas, inclusivas y participativas.

Otra recomendación para futuras investigaciones, en el caso de Colombia, sería analizar la relación entre la violencia escolar y, la influencia y accionar de los grupos armados ilegales en el marco del posconflicto, pues de la forma como se asuman por parte de la sociedad estos cambios en el conflicto armado interno, dependerá la formación en derechos humanos de los estudiantes de educación media, pues son susceptibles de verse influenciados por posturas contrarias a la defensa de los derechos fundamentales.

\section{REFERENCIAS}

Albor Chadid, L. I. \& Villamil López, L. (2012). Una mirada a las prácticas pedagógicas de los docentes en colegios públicos de Barranquilla y Girón (departamento de Santander) para intervenir los problemas de violencia escolar. Revista Educación y Humanismo, 14(23), 197-215. Recuperado de: http://publicaciones.unisimonbolivar.edu.co/rdigital/ ojs/index.php/educacion/article/view/723/745

Cataño, J. M. (2014). Caracterización de las prácticas de convivencia escolar en la educación básica primaria. Trabajo de Maestría. Universidad 
Pontificia Bolivariana, Medellín. Recuperado de: repository.upb.edu. co:8080/jspui/bitstream/123456789/1971/1/Tesis\%20biblioteca\%20 Claudia\%20Cata\%C3\%B10.pdf

Correa, M., Jaimes, J., Cardozo, S. \& Mantilla, C. (2013). Estrategia para la disminución de violencias en la escuela en la ciudad de Bucaramanga. Revista Justicia, 18(24), 14-24. Recuperado de http://publicaciones.unisimonbolivar.edu.co/rdigital/ojs/index.php/justicia/article/ viewFile/136/138

Echeverri, A., Gutiérrez, R. A., Ramírez, C. M. \& Morales, S. A. (2014). Hacia una construcción del concepto de violencias escolares. Revista Colombiana de Ciencias Sociales, 5(1).

Eljach, S. (2011). Violencia escolar en América Latina y el Caribe: superficie y fondo. Panamá: Plan Internacional y Unicef. Recuperado de http://www. unicef.org/lac/violencia_escolar_OK.pdf

Gil, M. Y. (2013). Medellín 1993-2013: Una ciudad que no logra encontrar el camino para salir definitivamente del laberinto. En: What happens when Governments Negotiate with Organized Crime? Cases Studies from the Americas. Washington D.C., Estados Unidos: Woodrow Wilson International Center for Scholars. Recuperado de: https://www.wilsoncenter.org/sites/default/files/Gil\%20Ramirez\%20-\%20Colombia\%20 -\%20Paper_1.pdf

González, V., Mariaca, I. \& Arias, J. (2014). Estudio exploratorio del bullying en Medellín. Pensando Psicología, 10(17), 17-25. Recuperado de http:// revistas.ucc.edu.co/index.php/pe/article/view/776/794

Martínez, V. (2005). Conflictividad escolar y fomento de la convivencia. Revista Iberoamericana de Educación, 38, 33-58. Recuperado de http://www.iea.gob.mx/ocse/archivos/DOCENTES/20\%20CONFLICTIVIDAD\%20ESCOLAR\%20Y\%20FOMENTO\%20A\%20LA\%20CONVIVENCIA.pdf

Ministerio de Educación Nacional (2013). Guías pedagógicas para la convivencia escolar. Sistema Nacional para la Convivencia Escolar. Recuperado de: http://www.mineducacion.gov.co/1759/articles287822_archivo_pdf.pdf

Ministerio de Educación Nacional. Decreto 1965 de 2013. Por el cual se reglamenta el Sistema nacional de convivencia escolar y formación para el ejercicio de los derechos humanos, la educación para la sexualidad y la prevención y mitigación de la violencia escolar. Recuperado de: http://www.mineducacion.gov.co/1621/articles-328630_archivo_pdf_ Decreto_1965.pdf

Ministerio de Educación Nacional. Ley 1620 de 2013. Por la cual se crea el Sistema nacional de convivencia escolar y formación para el ejercicio de los derechos humanos, la educación para la sexualidad y la prevención y mitigación de la violencia escolar. Recuperado de: http:// www.mineducacion.gov.co/cvn/1665/articles-319679_archivo_pdf.pdf

Organización de las Naciones Unidas (ONU) (2014). ¿Qué son los derechos humanos? Recuperado de http://www.ohchr.org/SP/lssues/Pages/ WhatareHumanRights.aspx 
Policía Nacional de Colombia (2010). Delinquir no paga. Recuperado de https://www.medellin.gov.co/irj/go/km/docs/wpccontent/Sites/ Subportal\%20del\%20Ciudadano/Convivencia\%20y\%20seguridad/ Secciones/Plantillas\%20Gen\%C3\%A9ricas/Documentos/2010/ DOCUMENTO_FUERZA_JOVEN_ABRIL_29_DE_2010\%5B1\%5D.pdf

Romero, D. R. (2012). Representaciones sociales de la violencia escolar entre pares, en estudiantes de tres instituciones educativas públicas de Bogotá, Chía y Sopó, Cundinamarca. Tesis de Maestría. Universidad Nacional de Colombia. Facultad de Ciencias Humanas, Bogotá. Recuperado de http://www.bdigital.unal.edu.co/8054/1/4458559.2012. pdf

Tobón, C. V. (2014). Representaciones sociales de la violencia escolar en la IE Maestro Fernando Botero. Tesis de Maestría. Universidad de San Buenaventura. Facultad de Educación, Medellín. Recuperado de http:// bibliotecadigital.usbcali.edu.co/jspui/bitstream/10819/2271/1/Representaciones_Sociales_Escolar_Tobon_2014.pdf

UNESCO (2011). Poner fin a la violencia en la escuela: guía para docentes. Nueva York: Autor. Recuperado de http://unesdoc.unesco.org/ images/0018/001841/184162s.pdf

UNESCO (2012). Educación en Derechos Humanos en la escuela primaria y secundaria: guía de autoevaluación para gobiernos. Nueva York: Autor. Recuperado de http://www.ohchr.org/Documents/Publications/SelfAssessmentGuideforGovernments_sp.pdf

UNICEF (2011). Violencia escolar en América Latina y el Caribe. Superficie y fondo. Panamá: Autor. Recuperado de http://www.unic ef.org/lac/ violencia_escolar_OK.pdf

Villalba, J. (2016). La convivencia escolar en positivo. Revista Educación y Humanismo, 18(30), 92-106. DOI: http://dx.doi.org/10.17081/ eduhum.18.30.1324 